\title{
The maximum coefficient of performance of internally irreversible refrigerators and heat pumps
}

\author{
Mohand A Ait-Ali \\ Département Génie Mécanique, Ecole Nationale Polytechnique, BP 182, \\ Hassen-Badi, Alger 16100, Algeria Visiting Research Scientist, University of \\ Michigan MEAM Department, 2212 G G Brown Building, Ann Arbor, \\ MI 48109-2125, USA
}

Received 14 August 1995

Abstract. A class of irreversible refrigeration cycles is investigated to determine the maximum coefficient of performance in the heat pump mode and the refrigerator mode. For the purpose of generality and simplicity of the results, finite-time heat transfer in the condenser and evaporator is expressed in terms of arithmetic mean temperature differences. The generic source of internal irreversibility is measured by a single irreversibility factor which transforms the Clausius inequality into an equality to simplify the cycle model. These optimum cycle performances are obtained as closed form analytical expressions in which the irreversibility factor has been shown to be simply related to the ratio of the actual and endoreversible cycle coefficients of performance.

\section{Introduction}

Unlike the finite-time maximum power cycle, which is a problem with two degrees of freedom, the optimum endoreversible refrigeration cycle can be optimized with respect to only one free variable [1]. If this variable is chosen to be the refrigerant temperature ratio for instance, one of these temperatures or the ratio of heat transfer conductances has then to be specified. In the few published articles about endoreversible refrigeration cycles, the additional constraint required to bound the optimum solution has consisted of one of the following.

(i) Specify the heat rejection load [2] in order to maximize the coefficient of performance $\beta$ of the heat pump.

(ii) Specify the refrigeration load [3] in order to maximize the coefficient of performance (COP) of the refrigerator.

(iii) Specify the refrigerant operating temperature range as a parameter [4] in order to maximize the refrigeration power, the refrigeration load and the heat rejection load.

These endoreversible refrigeration cycle problems have been solved using the Newtonian heat transfer model and, respectively, a constant temperature approach in the heat exchangers, the method of the effectiveness number of transfer units (NTU) and an arithmetic mean temperature difference. Whether simple or more complex, these endoreversible cycle models do not incorporate the essential physics of internally irreversible cycles in order to produce

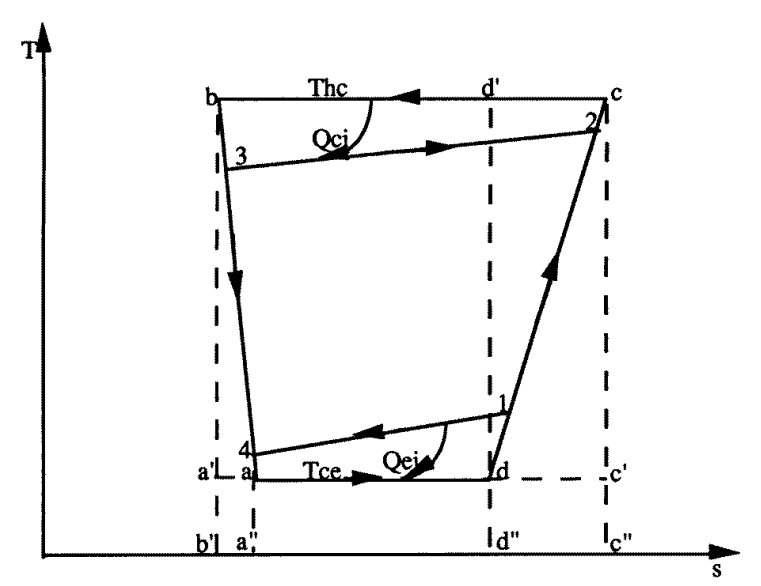

Figure 1. The irreversible refrigeration cycle $T-s$ diagram.

realistic performance predictions of refrigerators or heat pumps.

Internally irreversible refrigeration cycles are also problems with one degree of freedom since the particular form of the Clausius inequality constraint has no effect on the problem dimension. Considering an internally irreversible refrigeration cycle with a specified refrigerant operating temperature range and a generic source of entropy production, this author has shown [5] recently that the coefficient of performance is no longer a monotonically increasing function of the refrigeration temperature, as 
is the case for endoreversible cycles. However, this irreversible problem formulation could only lead to optimum numerical solutions whose analytical results would be more general and hence more useful.

It thus appears that a more realistic and yet still simple irreversible refrigeration model cycle is needed to predict the coefficient of performance of heat pumps and refrigerators better in more general terms. Improved predictions of performance parameters and equipment size are necessary for reliable estimates of investment and operating costs in preliminary cycle evaluations. The objective of this paper is to investigate the maximum COP of internally irreversible heat pumps and refrigerators using an irreversibility factor in the Clausius inequality in order to obtain analytical expressions for the optimum performance parameters.

\section{Formulation of the problem}

This problem formulation is based on the cycle $T-S$ diagram of figure 1 in which the heat source supplies the refrigeration heat load $Q_{e i}$ at a temperature varying from $T_{1}$ to $T_{4}$ in a counterflow evaporator and the heat sink receives the heat rejection load $Q_{c i}$, while its temperature increases from $T_{3}$ to $T_{2}$ in a counterflow condenser.

This refrigeration cycle is composed of the following processes.

(i) From $d$ to $c$ : a polytropic irreversible compression for which the net entropy production is equal to $s_{c}-s_{d}$; the time duration of this process is assumed to be negligibly small,

(ii) From $c$ to $b$ : an isothermal heat transfer for which the entropy decreases from $s_{c}$ to $s_{b}$ as a result of heat transfer from the condensing fluid into the heat sink, during a time $t_{c}$.

(iii) From $b$ to $a$ : a polytropic irreversible expansion for which the net entropy production is equal to $s_{a}-s_{b}$; the time duration of this process is assumed to be negligibly small.

(iv) From $a$ to $d$ : an isothermal heat transfer for which the entropy increases from $s_{a}$ to $s_{d}$ as a result of heat transfer into the evaporating fluid, during a time $t_{e}$.

In actual refrigeration cycles, condensation of the refrigerant occurs after some de-superheating, whereas evaporation is followed by some superheating prior to compression; these real heat transfer processes are therefore neither constant temperature, nor constant pressure ones. The isotherms considered here are averaged values, as would be obtained by taking the ratios of heat load to entropy change for each heat transfer process.

When this irreversible cycle is compared with the endoreversible cycle represented by processes $a^{\prime}-c^{\prime}-c-b-$ $a^{\prime}$, it is seen that the heat load $Q_{c i}$, rejected to the heat sink, is proportional to the rectangular area $b-c-c^{\prime \prime}-b^{\prime \prime}$ and the same for both cycles. However, the refrigeration load $Q_{e i}$, which is received from the heat source, is always less for the irreversible cycle. Consequently, the endoreversible refrigeration cycle always overpredicts coefficients of performance of actual refrigeration cycles in a manner which will be discussed in further detail when the main results are presented.

In this problem definition, arithmetic mean temperature differences are used as an upper bound approximation to logarithmic mean temperature differences (LMTD) to simplify algebra and yet obtain more realistic results than would be obtained with the constant temperature approach. Assuming the same minimum temperature approach $\delta$ in the condenser and evaporator, these arithmetic mean temperature differences are expressed by the following definitions:

$$
\begin{aligned}
& A_{m c}=\left(T_{h c}-T_{c}\right) / 2=x / 2 \\
& A_{m e}=\left(T_{e}-T_{c e}\right) / 2=y / 2
\end{aligned}
$$

with

$$
\begin{aligned}
& T_{c}=T_{0}-\delta \\
& T_{e}=T_{0}+\delta .
\end{aligned}
$$

Assuming heat conductances $\alpha_{c}$ and $\alpha_{e}$, respectively, for the condenser and the evaporator, the heat loads are expressed by

$$
\begin{aligned}
& Q_{c i}=\alpha_{c} x t_{c} / 2 \\
& Q_{e i}=\alpha_{e} y t_{e} / 2 .
\end{aligned}
$$

The sum of the heat transfer time durations in the condenser and evaporator is equal to the cycle time duration $T$, according to the following constraint:

$$
t_{c}+t_{e}=T
$$

Using the variable $z$ to define the relative heat transfer duration in the condenser and $1-z$ as the relative heat transfer duration in the evaporator, this constraint becomes

$$
z+(1-z)=1 \text {. }
$$

When the Clausius inequality between the entropy input to the evaporator and the entropy output from the condenser is written with an irreversibility factor $\kappa$, as was used in the optimization of internally irreversible heat engines [6,7], it takes the simple form

$$
\frac{-Q_{c i}}{T_{h c}}+\kappa \frac{Q_{e i}}{T_{c e}}=0 .
$$

The irreversibility factor $\kappa$ is greater than unity for an internally irreversible cycle and equal to unity for the endoreversible cycle; the larger this factor, the more irreversible the refrigeration cycle. This constraint shows that, for the same heat ratio $Q_{c i} / Q_{e i}$, the temperature ratio $T_{h c} / T_{c e}$ is smaller for the internally irreversible cycle.

\section{The maximum COP of an irreversible heat pump}

In this problem we seek the minimum power $P_{i}$ required to drive a heat pump for which the heat rejection load is specified as $P_{h}$; minimizing the required power for a given heat rejection load of a heat pump is equivalent to maximizing its coefficient of performance. 
Using the first law and the previously defined variables, the optimization problem is now written as

$$
\operatorname{Min} P_{i}=P_{h}-\alpha_{e} y(1-z) / 2
$$

subject to the specified heat rejection load

$$
\alpha_{c} x z /\left(2 P_{h}\right)=1
$$

and the irreversibility constraint

$$
\kappa\left(\alpha_{e} / \alpha_{c}\right)\left[\left(x+T_{c}\right) /\left(T_{e}-y\right)\right](y / x)(1-z) / z=1 .
$$

Solving for the product $\left(\alpha_{c} x z\right)$ from equation (11) and replacing into equations (10) and (12) reduces the constraints to only one, the variables being $y$ and $z$. Hence the problem has one degree of freedom.

Seeking the unrestricted extremum of the Lagrangian of the problem and eliminating the Lagrange multiplier from the two necessary conditions obtained with respect to $y$ and $z$ gives the following necessary condition for optimality:

$$
T_{e} / y=(1-z) \kappa\left[\left(\alpha_{e} / \alpha_{c}\right)+\left(\alpha_{e} / P_{h}\right) T_{c} z^{2}\right] / z^{2} .
$$

Eliminating $T_{e} / y$ from equations (12) and (13) yields the heat transfer time fractions as

$$
\begin{gathered}
z=\frac{t_{c}}{T}=\frac{1}{1+R} \\
1-z=\frac{t_{e}}{T}=\frac{R}{1+R}
\end{gathered}
$$

where $R$ is defined by the ratio

$$
R=\left[\alpha_{c} /\left(\alpha_{e} \kappa\right)\right]^{1 / 2} .
$$

It is seen that, as the irreversibility factor increases, the heat transfer time fraction increases in the condenser and decreases in the evaporator; this result is consistent with the condenser heat rejection load increasing relatively more than the frigeration load. It can be verified more easily from the Hessian of the unconstrained form of the objective function that this solution indeed corresponds to a minimum power requirement. Hence, the time fraction $z$ and the parameters to be obtained from it are optimum.

Solving for $x$ from equation (11) and then for $y$ from equation (13) leads to

$$
\begin{gathered}
x=2 P_{h}(1+R) / \alpha_{c} \\
y=\frac{2 P_{h} T_{e} R(1+R)}{2 P_{h}[(R+1)+1]+\alpha_{c} T_{c}} .
\end{gathered}
$$

The maxima of the refrigeration load are given by

$$
\frac{Q_{c i}}{T}=Q_{h}=\frac{P_{h} \alpha_{e} T_{e} R^{2}}{2 P_{h}[R(R+1)+1]+\alpha_{c} T_{c}} .
$$

The minima of the power input to the heat pump are given by

$$
P_{i}=P_{h}\left(1-\frac{P_{h} \alpha_{e} T_{e} R^{2}}{2 P_{h}[R(R+1)+1]+\alpha_{c} T_{c}}\right) .
$$

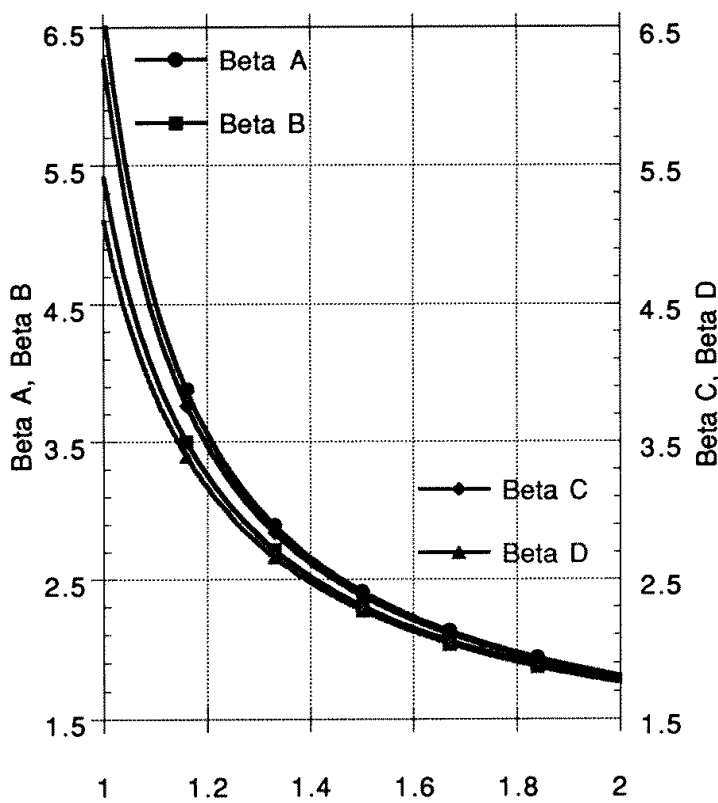

Figure 2. The heat pump coefficient of performance $\beta$ versus the irreversibility factor $\kappa$.

The maxima of the coefficient of performance of the heat pump are given by

$$
\beta=\frac{P_{h}}{P_{i}}=\left(1-\frac{P_{h} \alpha_{e} T_{e} R^{2}}{2 P_{h}[R(R+1)+1]+\alpha_{c} T_{c}}\right)^{-1} .
$$

The optimum coefficient of performance of the heat pump is seen to decrease with the specified heat rejection load $P_{h}$; that is, the smaller the heat pump requirement, the more efficient it would be. It also decreases with the temperature ratio $T_{e} / T_{c}$, which is more evident; but then, smaller heat pumps are expected to be less cost effective than larger ones.

The optimum arithmetic mean temperature differences in the condenser and evaporator are obtained by replacing for $x$ and $y$, respectively, in equations (1) and (2):

$$
\begin{gathered}
A_{m c}=\left(T_{h c}-T_{c}\right) / 2=P_{h}(1+R) / \alpha_{c} \\
A_{m e}=\frac{T_{e}-T_{c e}}{2}=\frac{P_{h} T_{e} R(R+1)}{2 P_{h}[(R+1)+1]+\alpha_{c} T_{c}} .
\end{gathered}
$$

The optimum ratio of these mean temperature differences is obtained as

$$
\frac{A_{m c}}{A_{m e}}=\frac{2 P_{h}[R(R+1)+1]+\alpha_{c} T_{c}}{R \alpha_{c} T_{e}} .
$$

The corresponding condensing refrigerant temperature is

$$
T_{h c}=T_{c}\left[1+2 P_{h}(R+1)\right] .
$$

The evaporating refrigerant temperature is

$$
T_{c e}=T_{e}\left(1-\frac{2 P_{h} R(R+1)}{2 P_{h}[R(R+1)+1]+\alpha_{c} T_{c}}\right) .
$$

The behaviour of the optimum coefficient of performance $\beta$ with respect to the irreversibility factor $\kappa$ is shown in figure 2 for two heat conductance ratios $\alpha_{c} / \alpha_{c}$ and the parameter $P_{h} / \alpha_{c}=10 \mathrm{~K}$. 


\section{The maximum COP of an irreversible refrigeration cycle}

In this problem, we seek the minimum power $P_{r}$ required to drive the refrigerator for which the refrigeration load is specified as $Q_{r}$; minimizing the required power for a given refrigeration load of a refrigeration cycle is equivalent to maximizing its COP. Using the first law and the previously defined variables, the optimization problem is written as

$$
\operatorname{Min} P_{r}=\alpha_{c} x z / 2-Q_{r}
$$

subject to the specified refrigeration load

$$
\alpha_{e} y(1-z) /\left(2 Q_{r}\right)=1
$$

and the irreversibility constraint

$$
\kappa\left(\alpha_{e} / \alpha_{c}\right)\left[\left(x+T_{c}\right) /\left(T_{e}-y\right)\right](y / x)(1-z) / z=1 .
$$

Solving for $y$ from equation (27) and replacing into equation (12) gives $x$ as

$$
x=\frac{2 \kappa Q_{r} \alpha_{e} T_{c}(1-z)}{\alpha_{c} \alpha_{e} T_{e} z(1-z)-2 Q_{r}\left[\alpha_{c} z+\kappa \alpha_{e}(1-z)\right]} .
$$

Replacing for $x$ in the objective function, we seek the unrestricted minimum of

$$
P_{r}=Q_{r}\left(\frac{\kappa \alpha_{c} \alpha_{e} T_{c}}{\alpha_{c} \alpha_{e} T_{e}-2 Q_{r}\left[\alpha_{c} /(1-z)+\kappa \alpha_{e} / z\right]}-1\right)
$$

or equivalently, the unrestricted minimum of the variable part of the denominator, or

$$
\phi=2 Q_{r}\left[\alpha_{c} /(1-z)+\kappa \alpha_{e} / z\right]
$$

for which the first derivative with respect to $z$ is zero with $z$ given by equation (14). The second derivative of $\phi$ with respect to $z$ is always positive, because

$$
\frac{\mathrm{d}^{2} \phi}{\mathrm{d} z^{2}}=4 Q_{r} \alpha_{e}\left[(z-1)^{-3}+z^{-3} \kappa \alpha_{e} / \alpha_{c}\right]>0 .
$$

Solving for $y$ from equation (28) then for $x$ from equation (29), gives

$$
\begin{gathered}
y=2 Q_{r}(1+R) / R \alpha_{e} \\
x=\frac{2 Q_{r} T_{c}(1+R)}{R^{2} \alpha_{e} T_{e}-2 Q_{r}(1+R)^{2}} .
\end{gathered}
$$

The maximum heat rejection load is obtained as

$$
\frac{Q_{h i}}{T}=Q_{h}=\frac{Q_{r} \alpha_{c} T_{c}}{R^{2} \alpha_{e} T_{e}-2 Q_{r}(1+R)^{2}} .
$$

The minimum power input to the refrigerator is obtained as

$$
P_{i}=Q_{r}\left(\frac{\alpha_{c} T_{c}}{R^{2} \alpha_{e} T_{e}-2 Q_{r}(1+R)^{2}}-1\right) .
$$

The maxima of the coefficient of performance of the refrigerator are given by

$$
\mathrm{COP}=\frac{Q_{r}}{P_{i}}=\left(\frac{\alpha_{c} T_{c}}{R^{2} \alpha_{e} T_{e}-2 Q_{r}(1+R)^{2}}-1\right)^{-1} .
$$

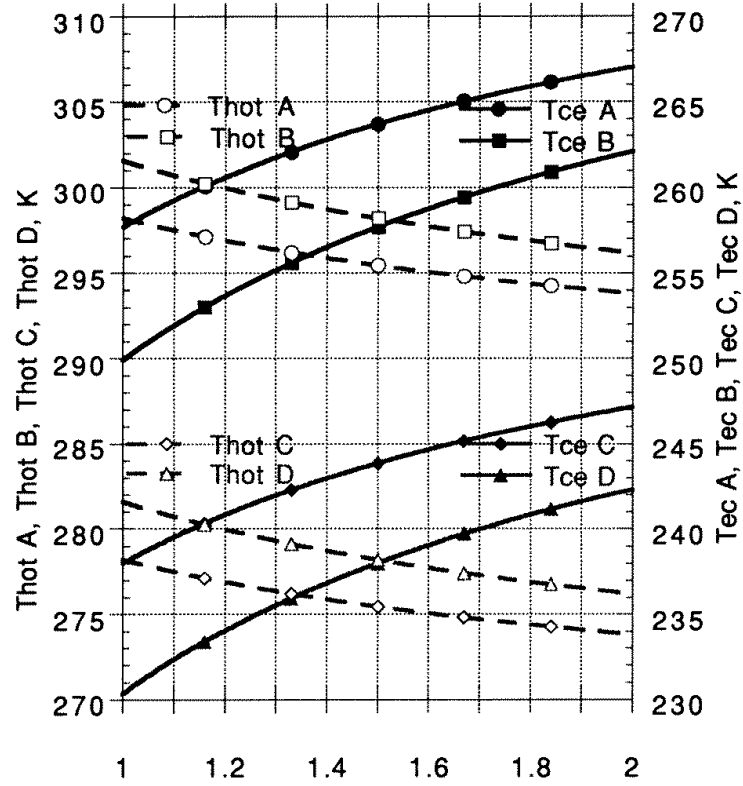

Figure 3. The heat pump exhaust and refrigerant evaporator temperatures versus the irreversibility factor $\kappa$.

The optimum coefficient of performance of the refrigerator is seen to decrease with the specified refrigeration load $Q_{r}$; that is, the smaller the refrigeration requirement, the less efficient the refrigeration cycle, just like in the heat pump case.

The optimum arithmetic mean temperature differences in the condenser and evaporator are obtained by replacing for $x$ and $y$, respectively, in equations (1) and (2):

$$
\begin{gathered}
A_{m c}=\frac{T_{h c}-T_{c}}{2}=\frac{T_{c} Q_{r}(1+R)}{\alpha_{e} T_{e} R^{2}-2 Q_{r}(1+R)^{2}} \\
A_{m e}=\frac{T_{e}-T_{c e}}{2}=Q_{r}(1+R) / R \alpha_{e} .
\end{gathered}
$$

The optimum ratio of these mean temperature differences is obtained as

$$
\frac{A_{m e}}{A_{m e}}=\frac{R \alpha_{e} T_{c}}{R^{2} \alpha_{e} T_{e}-2 Q_{r}(1+R)^{2}} .
$$

The evaporator refrigerant temperature is

$$
T_{c e}=T_{e}\left(1-\frac{2 Q_{r}(1+R)}{T_{e} R \alpha_{e}}\right) .
$$

The condenser refrigerant temperature is

$$
T_{h c}=T_{c}\left(1+\frac{2 Q_{r}(1+R)}{\alpha_{e} T_{e} R^{2}-2 Q_{r}(1+R)^{2}}\right) .
$$

The behaviours of the optimum coefficient of performance of the refrigerator and the refrigeration temperatures with respect to the irreversibility factor are shown in figures 4 and 5 , respectively.

\section{The relation between the efficiency and the irreversibility factor}

The irreversibility factor used here as a measure of refrigeration cycle internal inefficiency does not have an 


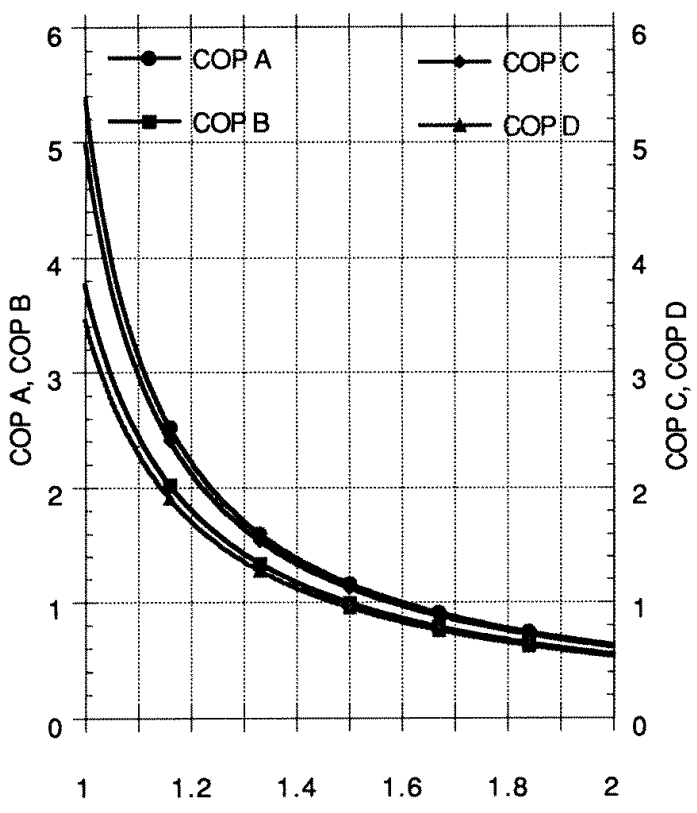

Figure 4. The refrigerator coefficient of performance versus the irreversibility factor $\kappa$.

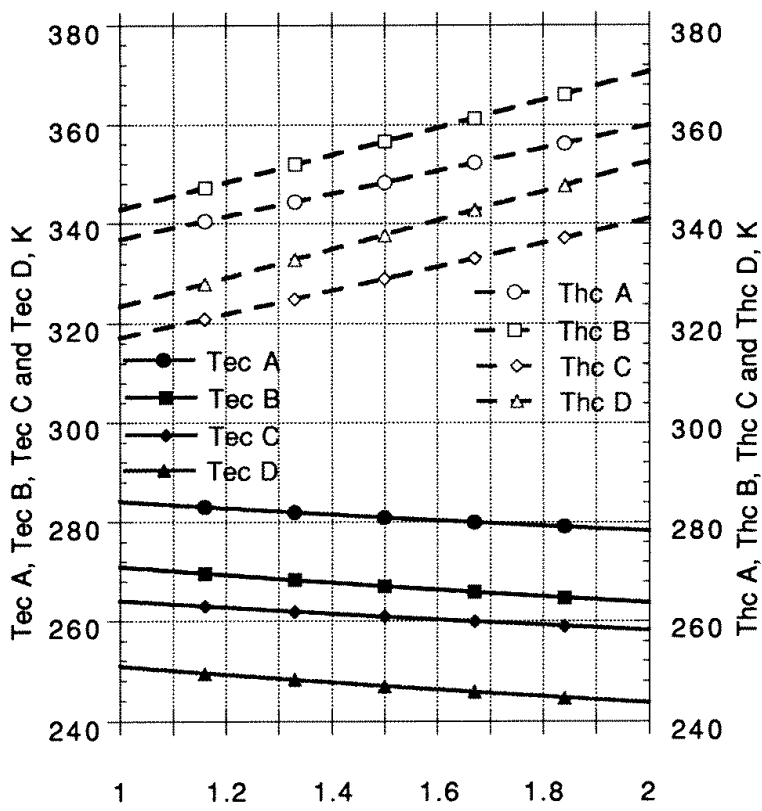

Figure 5. The evaporation and condensation temperatures versus the irreversibility factor $\kappa$.

operational significance like the isentropic efficiency of compressors. Comparing the endoreversible refrigeration cycle and the internally irreversible cycle by the ratio of their COP gives

$$
\frac{\mathrm{COP}_{i}}{\mathrm{COP}_{e}}=\frac{1-r}{\kappa-r}=\eta_{r}
$$

where $r$ is the temperature ratio $T_{c e} / T_{h c}$ and $\eta_{r}$ the compressor isentropic efficiency; it is assumed here that this ratio is a good measure of the isentropic efficiency of the compressor used to drive the actual refrigeration cycle.

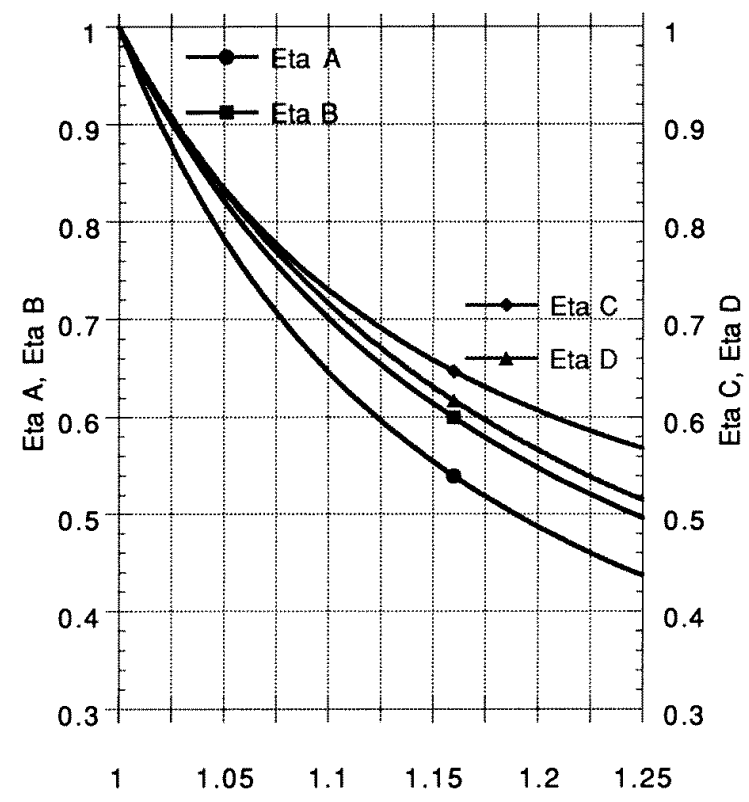

Figure 6. The heat pump endoreversible efficiency versus the irreversibility factor $\kappa$.

This isentropic efficiency thus decreases with increasing value of the irreversibility factor $\kappa$.

One can write similarly for the heat pump

$$
\frac{\beta_{i}}{\beta_{e}}=\frac{\kappa(1-r)}{\kappa-r}=\eta_{h}
$$

It is seen from these two equations that the heat pump efficiency is always greater than that of the refrigerator because their ratio is equal to $\kappa$ for the same cycle temperatures $T_{c e}$ and $T_{h c}$. These temperatures are consistent with respect to the $\kappa$ values and the values of the coefficients of performance obtained from the respective defining equations. These ratios of the coefficients of performance are given in figures 6 and 7, respectively for the heat pump and the refrigerator.

\section{A numerical example}

This example is intended to provide the discussion with some figures of merit; the refrigerator is considered for cooling atmospheric air to some temperature $5 \mathrm{~K}$ above the refrigerant temperature in the evaporator. The heat pump is considered for home heating from ambient air with an evaporator cold-end temperature approach of $5 \mathrm{~K}$.

The following numerical values are also used in the sample calculations and the figures presented. Two ambient temperature conditions are considered: 278.2 and $258.2 \mathrm{~K}$. Two condenser-to-evaporator heat conductance ratios are considered. These constitute the four cases labelled A, B, C and D.

(i) Heat pump $P_{h} /\left(\alpha_{c} T_{c}\right)=5$.

$T_{0}=278.2 \mathrm{~K}, \alpha_{c} / \alpha_{e}=1$ and 1.5 , respectively, for cases $\mathrm{A}$ and $\mathrm{B}$.

$T_{0}=258.2 \mathrm{~K}, \alpha_{c} / \alpha_{e}=1$ and 1.5 , respectively, for cases $\mathrm{C}$ and $\mathrm{D}$. 


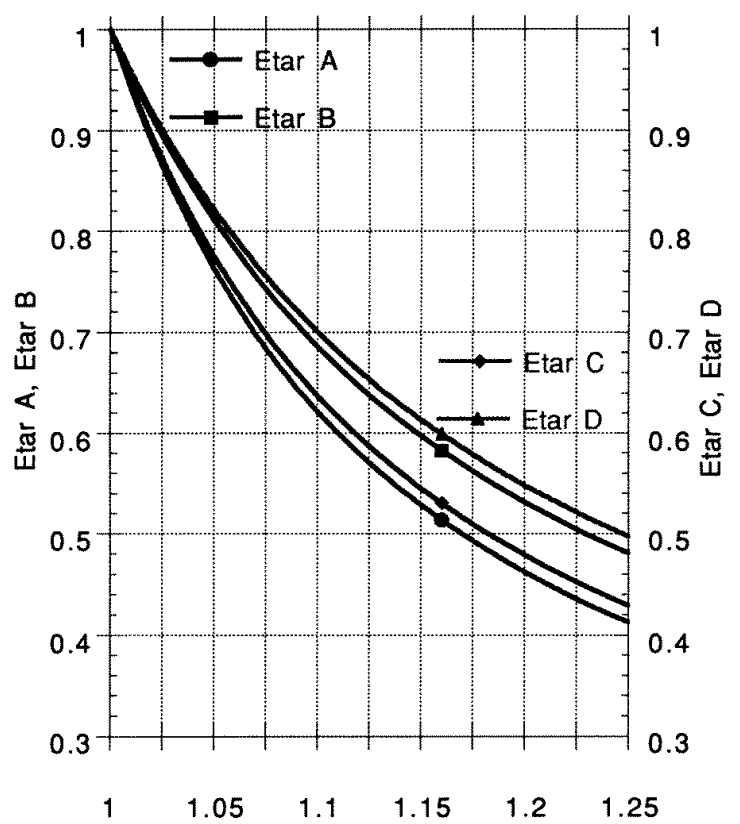

Figure 7. The refrigerator endoreversible efficiency versus the irreversibility factor $\kappa$.

(ii) Refrigerator $Q_{r} /\left(\alpha_{c} T_{c}\right)=5$.

$T_{0}=278.2 \mathrm{~K}, \alpha_{c} / \alpha_{e}=1$ and 1.5 , respectively, for cases $\mathrm{A}$ and $\mathrm{B}$.

$T_{0}=258.2 \mathrm{~K}, \alpha_{c} / \alpha_{e}=2$ and 3 , respectively, for cases $\mathrm{C}$ and $\mathrm{D}$.

The results are presented in figures $2-7$, bearing in mind that the range of practical significance for the irreversibility factor is $1-1.5$, the lower limit corresponding to the endoreversible heat pump. Figure 2 shows the heat pump coefficient of performance dropping rapidly from 6.5 to 2.7 when the irreversibility factor increases from 1 to 1.4 for case A. The lower value of the heat conductance ratio gives the higher coefficient of performance.

Figure 3 shows the air temperature delivered by the heat pump to decrease slowly with the irreversibility factor; the higher heat conductance ratio corresponds to the highest exhaust air temperatures. The refrigerant evaporator temperature increases with higher values of the irreversibility factor and smaller values of the heat conductance ratio.

Figure 4 shows that the behaviour of the refrigerator COP is similar to that of the heat pump. For the refrigerator, figure 5 shows that the behaviour of the refrigerant temperatures with respect to the irreversibility factor is opposite to that in the heat pump, whereas the effect of heat conductance on these temperatures is the same. Figures 6 and 7 show the variation in the endoreversible efficiency of the heat pump and refrigerator, respectively, with respect to the irreversibility factor.

\section{Conclusion}

A Carnot-like, internally irreversible refrigeration cycle is modelled using the Clausius entropy inequality with an irreversibility factor. It is optimized first for the maximum coefficient of performance of a heat pump, then for the maximum coefficient of performance of a refrigeration cycle with respect to the heat transfer time distribution in the condenser and evaporator. The choice of the heat conductance ratio appears to be critical in the heat pump case in order to avoid low refrigerant temperatures in the condenser and evaporator.

The optimum parameters are obtained as analytical expressions expressed in terms of the irreversibility coefficient, the heat conductance ratio and the temperature ratio. The effect of internal irreversibility is to decrease the condensation temperature and increase the evaporation temperature simultaneously in the heat pump mode, which leads to a lower coefficient of performance and lower heating temperatures. In the refrigerator mode, the coefficient of performance also decreases with respect to the irreversibility factor but under the effect of increasing condensation temperatures and decreasing evaporation temperatures of the refrigerant. In both modes, the choice of the heat conductance ratio and the ratio of power to condenser heat conductance appears to be critical to the performances sought.

\section{Acknowledgment}

The author gratefully acknowledges the support of the University of Michigan MEAM Department for the duration of this research.

\section{References}

[1] Leff H S and Teeters W O 1978 Am. J. Phys. 4619

[2] Blanchard C H 1982 J. Appl. Phys. 512471

[3] Klein S A 1992 Rev. Int. Froid 15181

[4] Ait-Ali Mohand A 1995 J. Appl. Phys. 774280

[5] Ait-Ali Mohand A 1995 J. Phys. D: Appl. Phys. to be published

[6] Özkaynak S, Göktun S and Yavuz H 1994 J. Phys. D: Appl. Phys. 271139

[7] Jincan Chen 1994 J. Phys. D: Appl. Phys. 271144 